\title{
Biomechanical analysis of PDMS channels using different hyperelastic numerical constitutive models
}

\author{
Cátia Cardoso ${ }^{a}$, Carla S. Fernandes ${ }^{\mathrm{a}, *}$, Rui Lima ${ }^{\mathrm{a}, \mathrm{b}, \mathrm{c}}$, João Ribeiro ${ }^{\mathrm{a}, \mathrm{d}}$ \\ a Instituto Politécnico de Bragança, ESTiG, Campus de Sta Apolónia, 5301-857 Bragança, Bragança, Portugal \\ ${ }^{\mathrm{b}}$ MEtRICs, Mechanical Eng. Dep., University of Minho, Campus de Azurém, 4800-058 Guimarães, Portugal \\ ' CEFT, Faculdade de Engenharia da Universidade do Porto (FEUP) Ruas Dr. Roberto Frias, 4200-465 Porto, Portugal \\ d Instituto Politécnico de Bragança, CIMO, Campus de Sta. Apolónia, 5300-253 Bragança, Bragança, Portugal
}

\section{A R T I C L E I N F O}

\section{Article history:}

Received 30 January 2018

Revised 13 April 2018

Accepted 13 April 2018

Available online 14 April 2018

\section{Keywords:}

Brain aneurysms

Displacement and strain fields

Numerical simulations

PDMS channels

\begin{abstract}
A B S T R A C T
Brain aneurysms are pathological dilatations of cerebral arteries and are known as one of the most common and serious cerebrovascular events. However, patients with cerebral aneurysms do not exhibit evident symptoms until they rupture. The main objective of the present study is to perform numerical characterizations of the biomechanical behavior of aneurysms in order to analyze the blood vessel wall behavior during the formation of an aneurysm. By taking into account different geometric and physiological parameters, flow simulations of a well-known Newtonian fluid (glycerin) was performed using the commercial finite volume method package Ansys ${ }^{\circledR}$ - Fluent, and pressure along the channel was determined. These pressures were imported into the channel, in the Ansys ${ }^{\circledR}$ - Static Structural, in order to evaluate and analyze the displacement and strain fields in the channel wall, caused by the internal pressure induced by the fluid flow. All calculations were performed by using the most widely accepted hyperelastic constitutive models and it was found that any constitutive model can be applied to this kind of studies, allowing to visualize where pressure achieves its maximum value and consequently, the most favorable region where the rupture is more likely to occur.
\end{abstract}

(c) 2018 Elsevier Ltd. All rights reserved.

\section{Introduction}

A cerebral aneurysm (or intracranial aneurysm) is the abnormal dilation, balloon or bulge out from the wall of a blood vessel located in the brain. This can be congenital or developed later in life due to the weakness of the arterial wall [1]. These aneurysms are responsible for $90 \%$ of aneurysms that occur at the points of Willis Circle branch at the base of the brain [2]. The pathogenesis of cerebral aneurysms is significantly influenced by the local hemodynamic environment. Most of the cerebral aneurysms formations result of areas subjected to in-creased hemodynamic pressures [3]. Therefore, it is important to understand the wall blood vessels behavior in the most susceptible areas to aneurysm rupture, as well as the factors that lead to the formation of such pathologies.

Blood vessels are soft tissues and exhibit a hyperelastic behavior [4] which is characterized to show a high deformation with an insignificant volume change and a very high nonlinearity of mechanical properties $[5,6]$. To analyze the mechanical behavior of blood vessels two approaches are usually used-in vitro exper-

\footnotetext{
* Corresponding author.

E-mail address: cveiga@ipb.pt (C.S. Fernandes).
}

imental studies [7-9] and numerical simulations [10-13]. In the former studies, silicone in vitro devices were developed [7-9] to mimic the biomechanical behavior of real blood vessels [14] or cadaveric arteries were used to analyze some mechanical properties [15]. Nowadays numerical simulations are gaining relevance in this field due to the development of hardware and software that allows accurate simulations of the mechanical behavior of blood vessels, with or without pathologies [16-20]. Nevertheless, despite the various works in this field, numerical modeling and experimental validation of the in vivo mechanical behavior of blood vessels still scarce in literature.

Recent algorithms are based on the classical hyperelastic behavior that corresponds to a mathematical description known as constitutive model. The constitutive model for a mechanical analysis is a relationship between the response of a body (for example, strain state) and the stress state due to the forces acting on the body, which can include the environmental effects. A wide variety of material behaviors are described with a few different classes of constitutive equations [21]. The hyperelastic models have been used extensively to model the non-linear behavior and anisotropic material, since soft tissues under large deformations often recover their elasticity. Note that, the constitutive behavior of hyperelastic materials is commonly defined in terms of deformation energy 
potential [22]. Some authors [23-25], have modeled wall's channels by means of hyperelastic models and have performed numerical studies to investigate the formation and growth of aneurysms in cylindrical channels and the elasticity of arterial tissue affected by Marfan's syndrome was analyzed by Merodio and Haughton $[26,27]$. However, most of the research works found in the literature have used only one constitutive hyperelastic model. To the best of our knowledge, evaluation and comparison studies by using different constitutive models have never been reported.

In previous works executed by Pinho et al. [7] and Rodrigues et al. [8] an in vitro system to mimic the biomechanical behavior of intracranial aneurysm (IA) was developed and measurements of displacement and strain field on bulge caused by a Newtonian fluid flow were made. The IA in vitro model used by Pinho et al. [7] was made of polydimethylsiloxane (PDMS) that is a biocompatible and hyperelastic polymer which is able to mimic the biomechanical behavior of the blood vessels [28]. More detailed information about the fabrication can be found in Pinho et al. [7]. Rodrigues et al. [8] have measured the displacement and strain fields caused by the fluid flow of a Newtonian viscous fluid (glycerin) on the wall of the IA. The measurements were carried out with a 3D digital image correlation system. Following the same line of research, the natural step would be to perform numerical simulations of the biomechanical behavior of the referred IA model. Hence, the main goal of this work is to analyze the biomechanical behavior of the IA model developed by Pinho et al. [7] and Rodrigues et al. [8] by using different constitutive hyperelastic models. The study was performed by applying the commercial software Ansys ${ }^{\circledR}$ that is used to model solids together with the Finite Element/Volume Method, in order to solve the problems in daily life modeling shapes / functions, stresses, strains, fluid flow, among others [29]. The Ansys-Fluent software is used for flow simulations and allows to obtain a better understanding of the fluid motion around the objects, as well as fluid behavior in complex circulatory system by conducting the fluid analysis [30]. The Ansys-Static Structural software is used to study the structural part of an element, in order to calculate and analyze the displacements and strains caused along the entire or a section of the structure. Thus it is possible to improve our understanding of the distortion that a structure will undergo, during the application of a force, pressure or other charge.

\section{Problem description}

In the present study, numerical simulations of biomechanical wall's behavior of an IA model used in previous experimental works [7,8], Fig. 1, were performed considering laminar Newtonian incompressible flow within the channel. Geometrical and material properties of the channel, as well as the operation conditions and the working fluid, were chosen in order to reproduce the experimental work performed by Rodrigues et al. [8].

However, the fabrication of this aneurysm model is difficult and has low repeatability. Rodrigues et al. [8] have found that the main limitations of the tested in vitro aneurysm model were mainly related to the irregular wall thickness and surface roughness of the inner channel. New fabrication methodologies able to overcome most of these limitations are currently under development.

\subsection{Fluid flow}

Simulation of the inner flow was performed in order to determine the pressure field in the inner wall of the channel, which was used as a boundary condition in the static structural modeling. For that, the computational domain was confined to the interior of the channel presented in Fig. 1 and can be observed in Fig. 2.

Simulation of the fluid flow was performed for $60 \%$ of glycerin and $40 \%$ of pure water, i. e., the working fluid used by Rodrigues

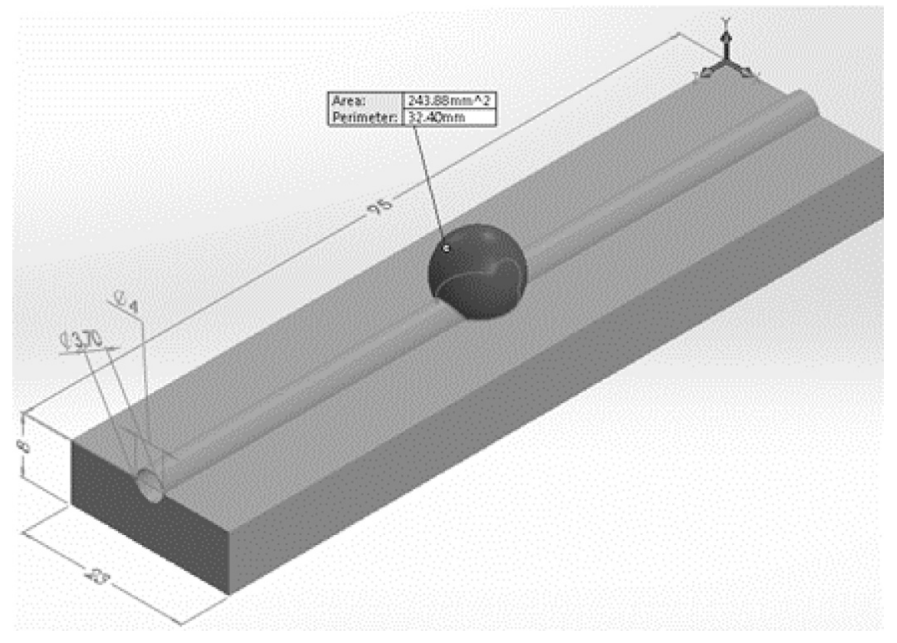

Fig. 1. Representation of studied in vitro channel.

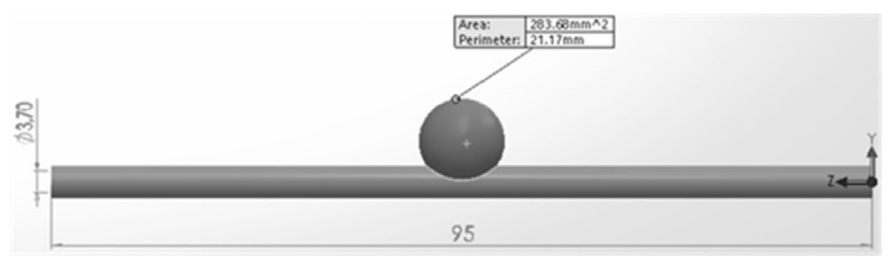

Fig. 2. Representation of the channel to perform flow simulations.
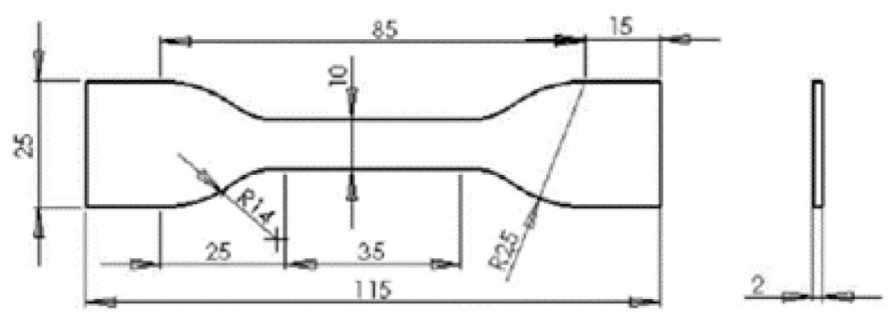

Fig. 3. Geometry and dimensions (in $\mathrm{mm}$ ) of the specimen.

et al. [8]. The properties of this fluid are well known and have enough viscosity to measure the pressure drops. For a temperature of $20^{\circ} \mathrm{C}$, the properties of this fluid are: density $(\rho)$ of $1153.8 \mathrm{~kg} / \mathrm{m}^{3}$ [31] and viscosity $(\eta)$ equal to $1.08 \times 10^{-2}$ Pa s [32].

\subsection{Structural analysis}

The studied channel, represented in Fig. 1, is made in polydimethylsiloxane (PDMS) which the commercial name is Sylgard® 184 from Dow Corning Corporation and intends to reproduce the IA model used by Rodrigues et al. [8] in their experimental studies.

In numerical simulations, wall hyperelastic properties behavior was described by the different constitutive models presented below.

The Mooney-Rivlin model is used to model the mechanical behavior of the soft biological tissue when the test material is hyperelastic [33]. The classic formula of strain energy of the MooneyRivlin model for incompressible hyperelastic materials it is often used in behavior description of incompressible isotropic materials, rubber type and soft tissue [33]. The soft biological tissues and rubber may be characterized as highly non-linear materials subjected to finite strains [33]. 


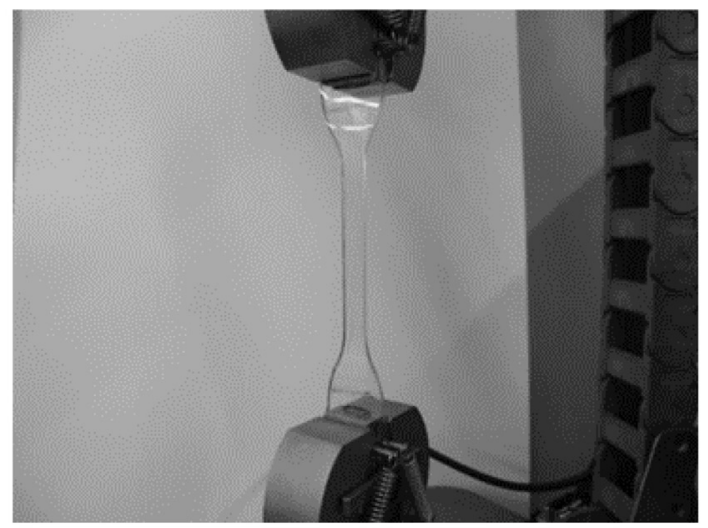

(a)

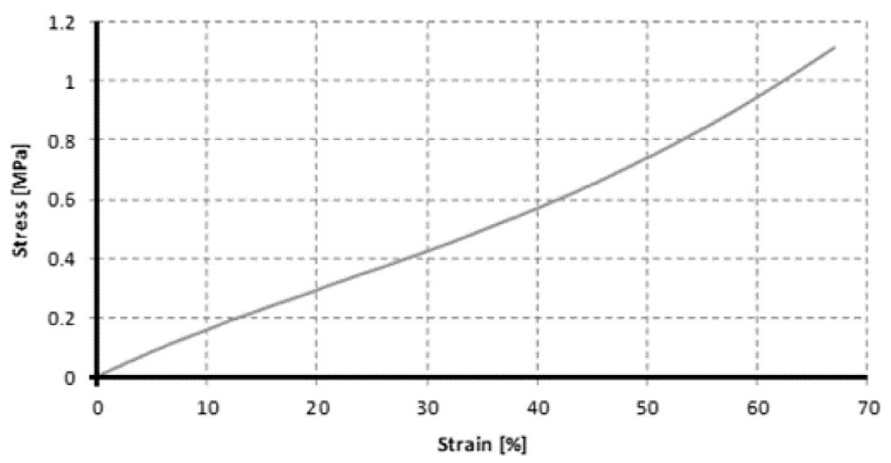

(b)

Fig. 4. Tensile test: (a) detail of the tensile test; (b) example of a stress-strain curve obtained by a tensile test.

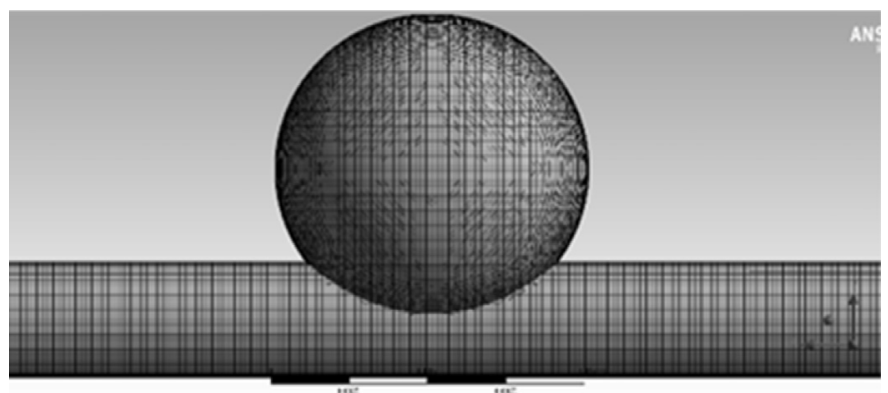

Fig. 5. Mesh used in the computational fluid dynamic calculations.

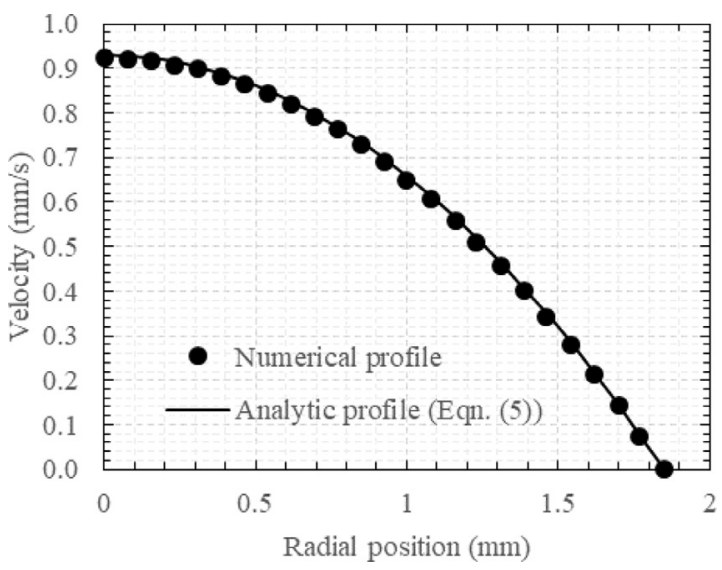

Fig. 6. Velocity profile in the region before the aneurysm.
The constitutive law Mooney-Rivlin derived hyperelastic incompressible material is described from the following equation [33]:

$\psi=C_{10}\left(I_{1}-3\right)+C_{01}\left(I_{2}-3\right)$

where $\psi$ is the strain energy density function, $I_{1}$ and $I_{2}$ are the constant strain and $C_{10}$ and $C_{01}$ are the material constants [33,33]. The Mooney-Rivlin coefficients $C_{10}$ to $C_{01}$ can usually be independently determined by suitable adjustment of a load deflection curve [34].

The Ogden model, Eq. (2), describes the changes of the main extensions of a reference to the current configuration. This plays a crucial role in the theory of finite elasticity [33]. The model Ogden ("2 times N"-parameter) can be described as follows [34]:

$\psi=\sum_{n=1}^{N} \frac{\mu_{n}}{\alpha_{n}}\left(\lambda_{1}^{\alpha_{n}}+\lambda_{2}^{\alpha_{n}}+\lambda_{3}^{\alpha_{n}}-3\right)$

where $\alpha_{n}$ and $\mu_{n}$ are the material constants, $N$ the number of terms in the series and $\lambda_{i}$ the three different relationships of the principal forces [34]. The rubber Ogden model may describe incompressible and almost incompressible behavior, in contrast to the Mooney-Rivlin model [34].

The Yeoh model of hyperelastic materials proved to be popular because it depends only on the first invariant deformation, $I_{1}$, and it has been satisfactorily used for various forms of deformation model based only on data obtained from a uniaxial tensile test. However, this model has shown to be inaccurate to calculate and model small deformations [35].

The shape of the strain energy density Yeoh function is given by [34]:

$\psi=\sum_{i=1}^{3} C_{i 0}\left(I_{1}-3\right)^{i}$

where $I_{1}$ is the constant strain and $C_{i 0}$ the material constants [36].

Among the strain-invariant based models, a polynomial form of energy density relation proposed by Rivlin (1948) is the first and the commonest one. Eq. (4) depicts the general polynomial form with $C_{i}$ as material parameter [37,38].

$\psi=\sum_{i=1}^{N} C_{i}\left(I_{1}-3\right)^{i}$

The polynomial and reduced polynomial models are therefore generalizations of a number of other functions. The Mooney-Rivlin model was introduced as one specific case of the polynomial form (Mooney, 1940); it was also studied by Rivlin in a series of papers on large elastic deformations [38].

\section{Experimental tests}

The structural numerical simulations were taken into account the stress-strain curves obtained by the PDMS tensile tests. To perform these tests the specimens which have the geometry in agreement with the BS 2782 standard was previously manufactured. In Fig. 3 the dimensions of specimen are shown.

The PDMS used in this study was the Dow Corning's Sylgard $184^{\circledR}$ and it was supplied in a kit composed by a prepolymer and a curing agent which need to be mixed depending on the desired bulk ratio. In this particular case, $1 \mathrm{~g}$ of curing agent was added to $10 \mathrm{~g}$ of prepolymer, i. e., ratio 10:1. The prepolymer of PDMS and the curing agent were then mixed with a spatula for about $5 \mathrm{~min}$ and then placed in a desiccator which was connected to a vacuum pump to remove all existing air bubbles. This mixture was poured into a mold with the geometry of the specimen. Finally, the mold with the PDMS was placed into an oven for one hour at $80^{\circ} \mathrm{C}$.

Three tensile tests were performed in an Instron ElectroPuls E1000 machine, with a $2 \mathrm{kN}$ load cell, in displacement control, 


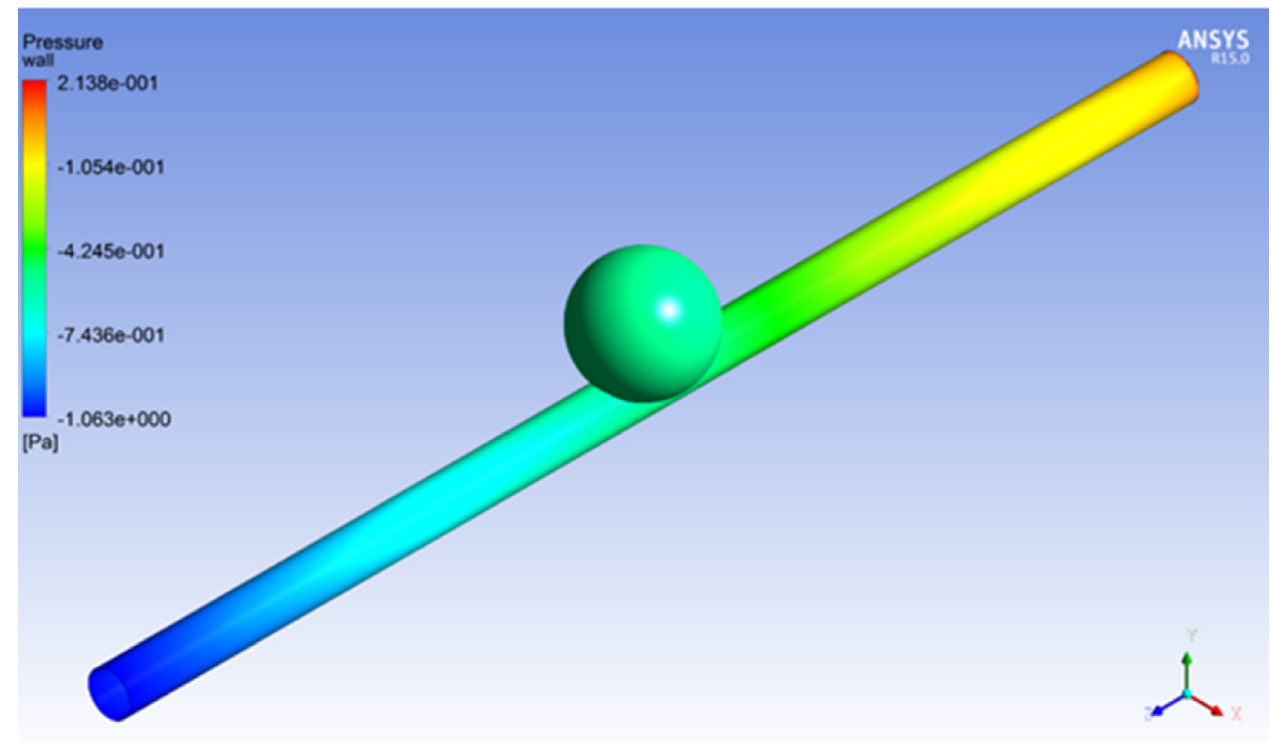

Fig. 7. Static pressure field at the wall of the channel (main flow direction is the positive $z$-axis).

up to, approximately, $60 \%$ of strain with a displacement rate of $5 \mathrm{~mm} / \mathrm{min}$. The used universal test machine has designed for dynamic and static testing on a wide range of materials and components. In these experimental tests, the room temperature was around $25{ }^{\circ} \mathrm{C}$ and the relative humidity was about $20 \%$. Initially the specimens were marked by making two lines in the perpendicular direction of the specimen axis with $85 \mathrm{~mm}$ between them by following the standard recommendations. After mounting the specimen in the universal tensile machine, it was first verified the alignment of the specimen relatively to the test direction and then a pre-load of $1 \mathrm{~N}$ was applied. Note that, we have used sandpaper within the grips in order to minimize the slip effect between the specimen surface and the contact surface of grips. Each test was repeated twice to verify any possible error and the repeatability of the experimental test.

In Fig. 4 it can be observed the test of a hyperelastic material and an example of a stress-strain curve obtained by a tensile test.

\section{Numerical simulations}

\subsection{Flow analysis}

As referred before, the first step of the numerical study performed in this work was to calculate the pressure field in the interior part of the wall channel represented in Fig. 1. The governing equations, mass and momentum conservation equations for an incompressible Newtonian fluid flow, were solved using the finite volume commercial software package Ansys-Fluent.

The discretization of the geometrical domain was based on CutCell method, with an element size equal to $0.25 \mathrm{~mm}$ having a total of 352,045 nodes and 344,508 elements (Fig. 5).

As boundary conditions, no slip at the wall was imposed and a constant velocity of $0.46 \mathrm{~mm} / \mathrm{s}$ was imposed in the inlet.

In order to validate the computational fluid dynamic calculations, two tests in the region before the aneurysm were performed. First, velocity profile was compared with the analytical solution for a Newtonian fully developed flow in a circular pipe which can be determined by [39]:

$v(r)=2 u\left(1-\left(\frac{r}{R}\right)^{2}\right)$ where $v$ is the velocity, $u$ the mean velocity, $R$ the radius of the channel and $r$ the radial position.

In Fig. 6, it is possible to verify the good agreement between numerical results and analytic profile (mean deviation of $1 \%$ ), which allow us to conclude that numerical model predicts well the local properties of the studied flow. Additionally, the pressure drop obtained was compared with the one predicted by HaggenPoiseuille equation [40]:

$\Delta P=\frac{8 \eta L u}{R^{2}}$

where $\Delta P$ represents the pressure drop, $\eta$ the fluid viscosity and $L$ the channel length.

Once again, a good agreement between pressure drop obtained by Eq. (6) $(\Delta P / L=11.741 \mathrm{~Pa} / \mathrm{m})$ and numerical result $(\Delta P / L=11.739 \mathrm{~Pa} / \mathrm{m})$ was found (relative error of $0.02 \%$ ).

Since numerical calculations revealed good predictions for both local and global properties of the studied flow, we can conclude that pressure field in the wall is also correct and can be observed in Fig. 7.

\subsection{Structural analysis}

After calculating the pressure caused by the fluid (Fig. 7), study of the mechanical behavior of the channel wall was performed using the Ansys ${ }^{\circledR}-$ Static Structural in order to analyze displacement and strains.

To calculate the strains and displacements that occurred on the channel's wall for each simulation, different constitutive models were applied in order to evaluate the most suited parameters to this type of study. During the pre-processing step each constitutive model was solved by following the curve fit based on stress-strain points obtained experimentally. As an example, the approximation of stress/strain curve by the Mooney-Rivlin 5 Parameter model is presented in Fig. 8. This procedure is repeated for each constitutive model investigated in this work.

The channel used in this part of the study (Fig. 1) was discretized by elements of $0.90 \mathrm{~mm}$, resulting in a mesh with 42,898 nodes and 24,045 elements, Fig. 9. After mesh generation, the constitutive model was established where the base was fixed on the model and the pressure (calculated from Fluent) was imported to the static structural as boundary condition. 


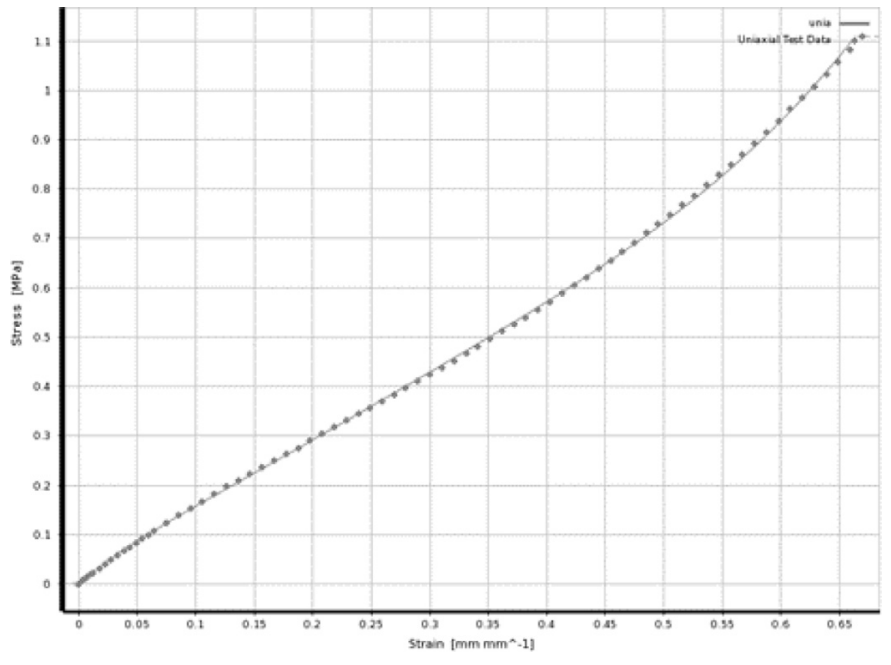

Fig. 8. Stress-strain curve for Mooney-Rivlin 5 Parameter model.

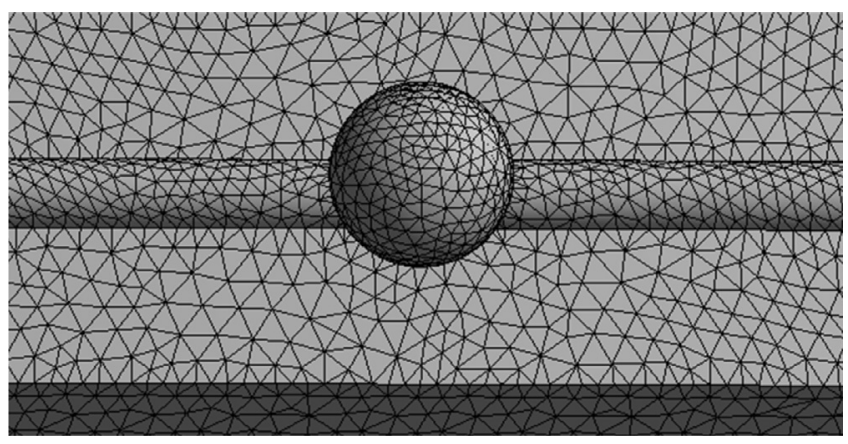

Fig. 9. Mesh used for the structural analysis.

For this kind of nonlinear structural analyses the large displacement option must be used. Since these analyses can be challenging to solve, a convergence criterion must be defined. In the present study it was used the Newton-Raphson Residuals to diagnose whether the solution tends to convergence. In this situation of non-convergence, the Newton-Raphson Residuals show which areas have high residual forces.

\section{Results and discussion}

Regarding the mechanical study, the eight constitutive models presented before was used to describe the properties of the wall: Ogden 2nd and 3rd Order, Yeoh 2nd and 3rd Order, Mooney-Rivlin 5 and 9 Parameter and Polynomial 2nd and 3rd Order. Although all models have converged, the results have showed significant differences.

In Figs. 10 and 11, the strain and displacement fields obtained by computational simulation for the model of Polynomial 3rd Order and Yeoh 2nd Order are shown.

Fig. 10 corresponds to the total displacement occurring in the channel wall for these two constitutive models, i. e., Polynomial 3rd Order and Yeoh 2nd Order. From these figures, we can conclude that although the maximum and minimum values of the displacements are in the same region, the model Yeoh 2nd Order shows higher values than the polynomial. The same applies to the equivalent elastic strain shown in Fig. 11.

The maximum and minimum strain are in the same region of the channel for all models, which makes them consistent results among themselves and close to other authors, namely Bazilevs et al. [41] and Carty et al. [13].

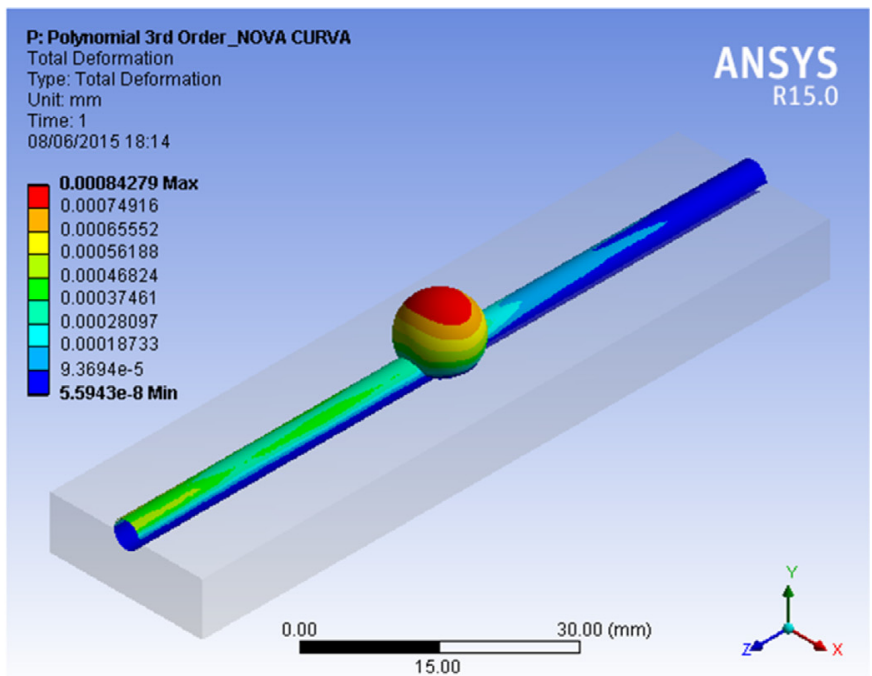

(a)

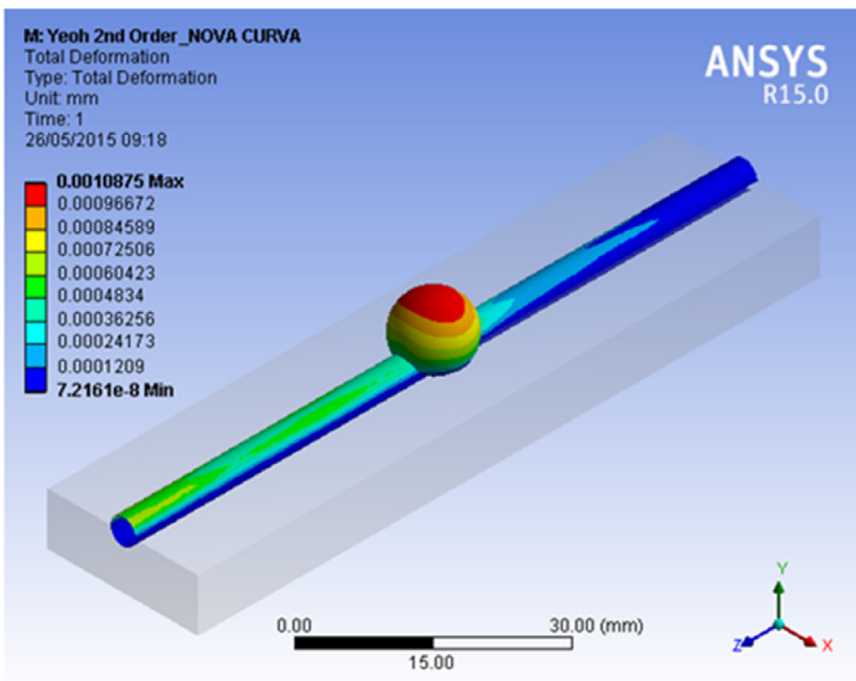

(b)

Fig. 10. Displacements for different kinds of models: (a) polynomial 2nd order; (b) Yeoh 2nd order.

After strain and displacement analysis across the channel, some points along the aneurysm dome were analyzed, according to the $z$ direction (fluid flow direction) and the $x$ direction, Fig. 12, in order to have a better perception of the differences between the different constitutive models and observe the displacements and strains occurring in this area. In both directions, the points were marked on the right side to the left side.

The values of displacement and strain for the points shown in Figs. 10 and 11 were calculated, using the nine studied constitutive models, to analyze a more accurately the values at these points of the aneurysm and also to facilitate the observation of the variation of the calculated results with the different constitutive models. In Figs. 13 and 14 the strains along the $z$-axis and $x$-axis, respectively, are presented.

From Fig. 13 it is possible to observe that along the $z$-axis, the strain values decrease as it approaches to the center of the aneurysm dome, reaching values close to zero. Then, the values increase up to values of about $80 \mu$ strain and at locations close to 


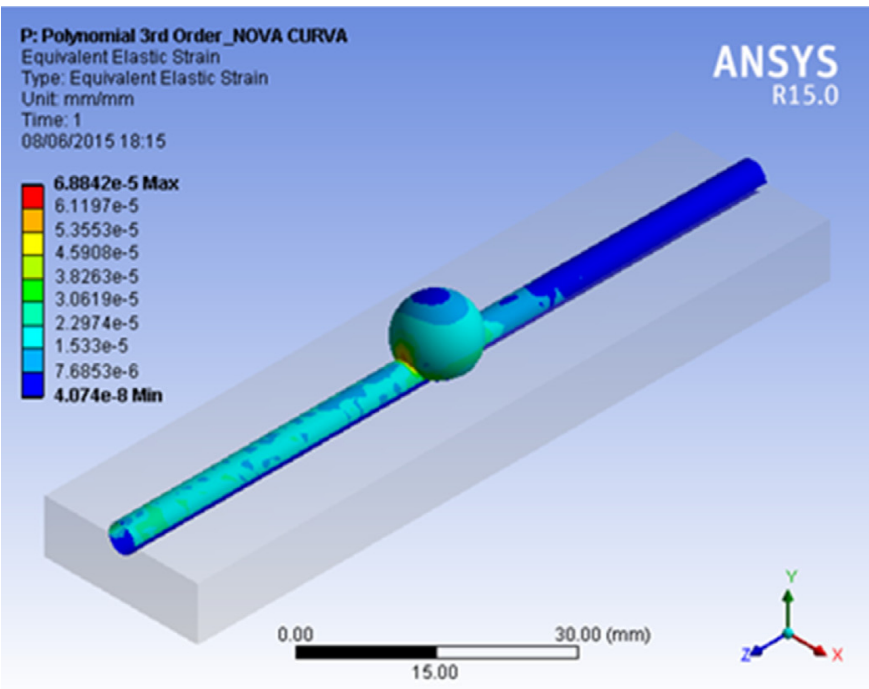

(a)

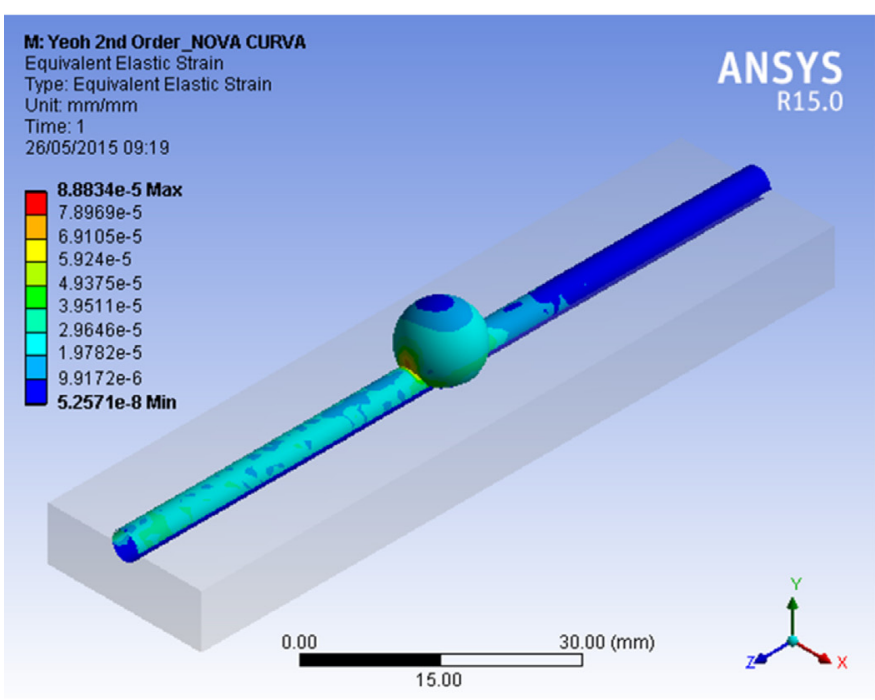

(b)

Fig. 11. Strains for different kinds of models. (a) Polynomial 2nd order; (b) Yeoh 2nd order.

the channel intersection the strain tends to decrease down to values between 70 and $60 \mu$ strain.

Results presented in Fig. 13 show that the maximum values are found close to the transition regions around the intersection of the main channel with the aneurysm.

Regarding Fig. 14, the strains begin to increase until the height of the channel, starting to decrease thereafter until a value close to zero is reached at the center of the aneurysm increased again until the channel height suffers a slight decrease of the deformation values.

In Figs. 15 and 16, displacements along the $x$-axis and $z$-axis, respectively, can be observed.

Overall, it is possible to conclude that the displacements are increasing toward the top of the aneurysm, having maximum values in both directions $\left(x\right.$ and $z$ ), of approximately $12 \times 10^{-3} \mathrm{~mm}$ for the model Yeoh $2^{\text {nd }}$ Order, that is the model that has higher values. In contrast the constitutive model of polynomial $3^{\text {rd }}$ order has the lower value of $8.0 \times 10^{-3} \mathrm{~mm}$.

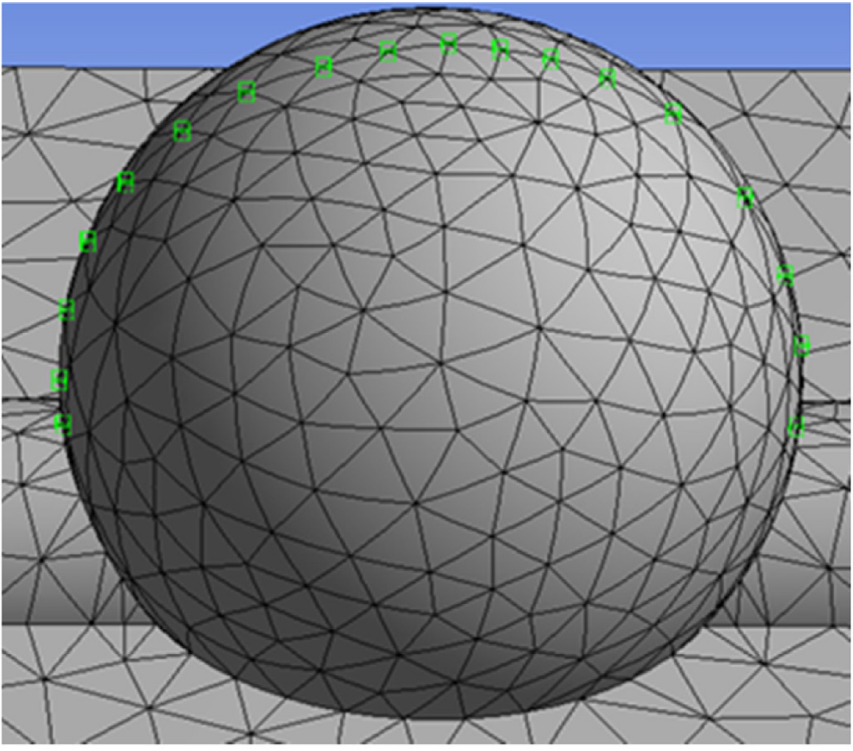

(a)

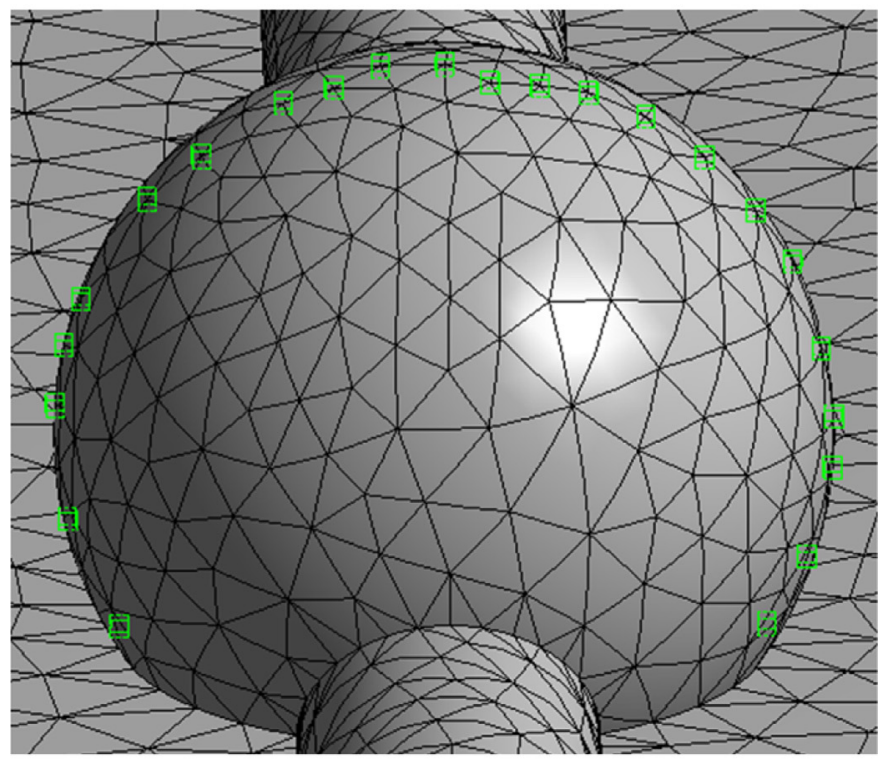

(b)

Fig. 12. Points in the aneurysm where displacements and strains were determined: (a) in the $z$ direction; (b) in the $x$ direction.

Additionally, Figs. 13-16 have shown that, qualitatively, the constitutive models studied in this work have led to similar fields of displacements and strains. However, when quantitative displacements and strains values are examined in more detail, some differences can be found.

It can also be seen that the Mooney-Rivlin 5 parameter and the Polynomial 2nd order models have exactly the same values for the displacements and strains. The model which exhibits lower displacement and strain values is the Polynomial 3rd order and the higher values is the Yeoh 2nd order.

These numerical simulations show the mechanical behavior of aneurysm models. However, it is important to identify some limitations of this analysis, namely, the simplicity of the aneurysm model, the simulated material could not totally mimic the tissues of blood vessels because these material have anisotropic proper- 


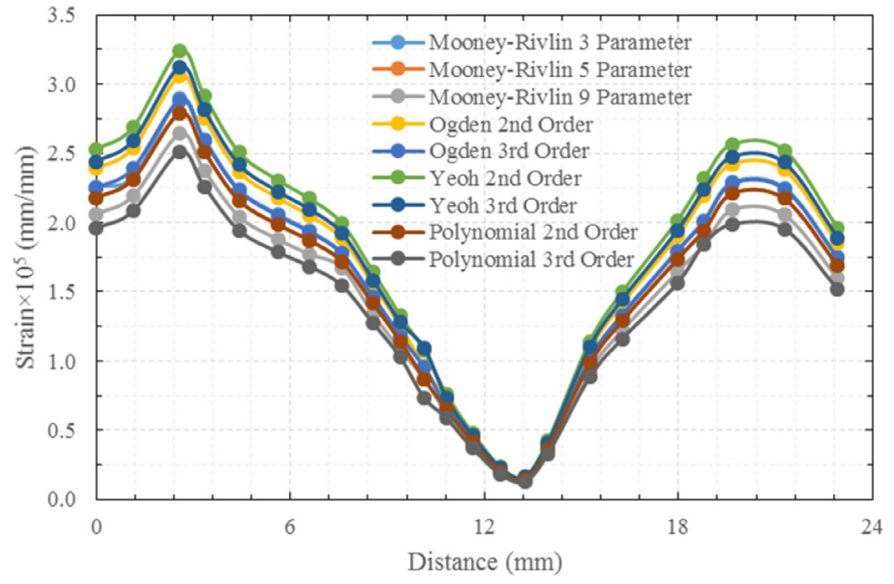

Fig. 13. Strain on the aneurysm along the $z$-axis.

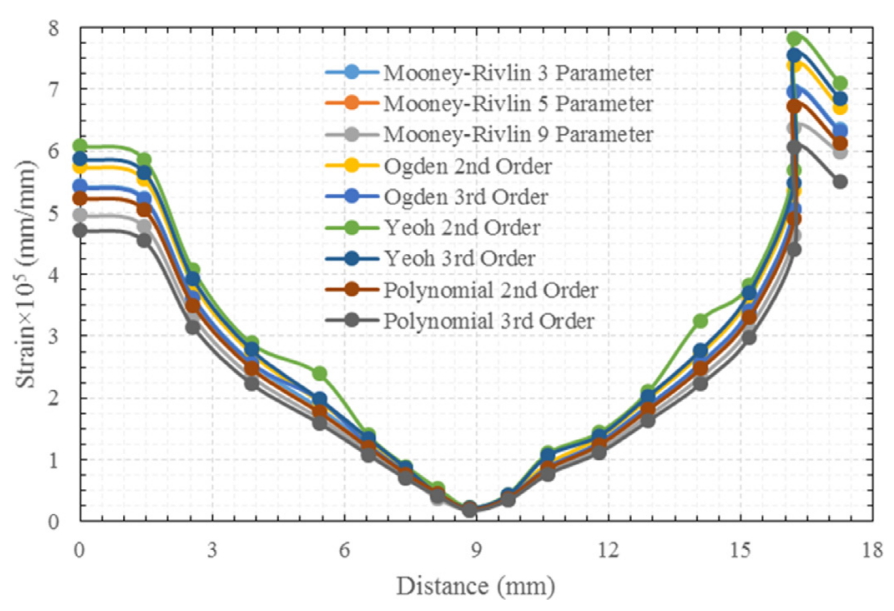

Fig. 14. Strain on the aneurysm along the $x$-axis.

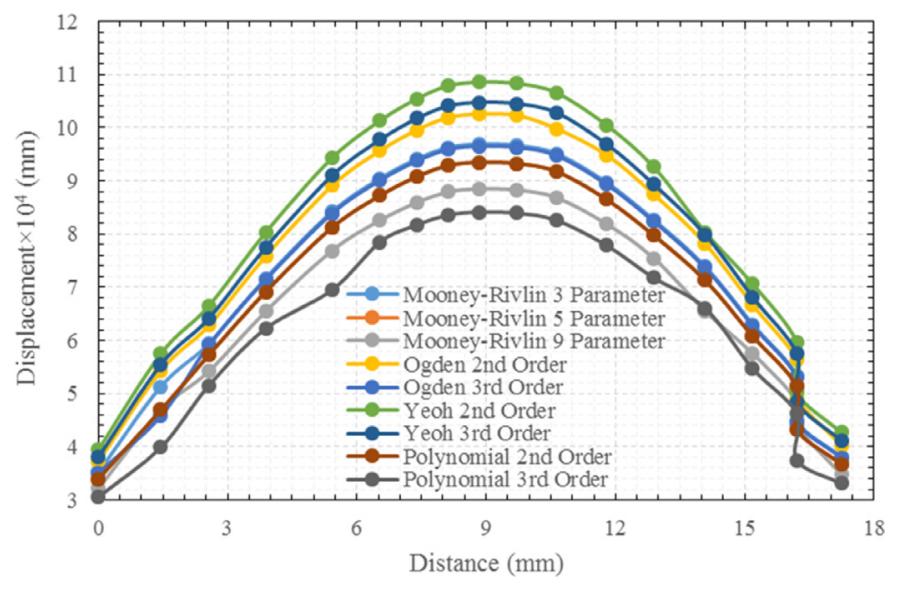

Fig. 15. Displacement on the aneurysm, along the $z$-axis.

ties while the PDMS is an isotropic material. However, this limitation not changed substantially the detection of risky zones. Other important limitations are related with the used fluid which have different properties from the blood and the fluid flow of humans is pulsatile while stationary simulations were performed in this work. Another factor that was not considered in this analysis was the effect of residual stresses. The residual stresses are stresses that exist in the body in the absence of external loads and change the response body when subjected to external charging, these arise from the manufacturing processes. There are many studies

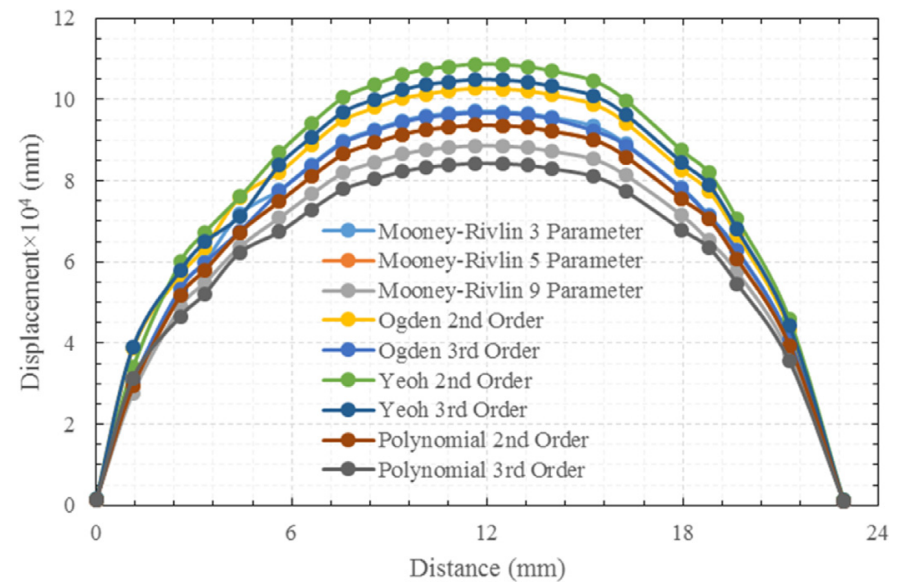

Fig. 16. Displacement on the aneurysm, along the $x$-axis.

of residual stresses effect in mechanical behavior of linear elastic material, typically metals, however only few authors [42] reported this kind of analysis applied to materials with non-linear behavior. An important study was done by Merodio et al. [42], these researchers studied the effect of residual stresses on finite deformation of elastic materials and they verified that for the inflation of a tube with negative residual stresses the material is more deformable when comparable with the situation in the absence of residual stresses. Applying this principle to the present work, if residual stresses were considered a lower pressure was required to achieve the same level of displacement.

\section{Conclusions}

The present study shows that the different kinds of hyperelastic constitutive models tested in this work can be applied to study the behavior of a PDMS wall of an IA in vitro model, particularly for the analysis of stress and displacement field on the aneurysm. Overall, the obtained results have shown qualitatively good agreement. This fact leads us to assume that the approach used in the present work is appropriate to describe the biomechanical behavior of hyperelastic walls for the tested IA model and that could be successfully reproduced in more realistic models. However, further work needs to be performed as we have also observed some discrepancies between our displacement and strain values and other experimental studies.

All the tested models have the values of upper and lower displacements and strains in the same region, presenting important variations from one model to another. Overall, it was shown that the higher values of strain and displacement correspond to the constitutive model of Yeoh 2nd whereas the lower value happens for the polynomial 3rd order. It is worth mentioning, that the tested constitutive models can be applied to hyperelastic materials, such as soft tissue (blood vessels), which makes these models a promising way to perform simulations under more realistic conditions. The numerical simulation is an important tool to predict potential risky zones of rupture which could help in the development of more efficient stents and surgical procedures.

\section{References}

[1] Y. Lee, Hemodynamics of a cerebral aneurysm model, Bioengineering Graduate, University California, Berkeley, USA, 2006.

[2] M. Nabaei, N. Fatouraee, Computational modeling of formation of a cerebral aneurysm under the influence of smooth muscle cell relaxation, J. Mech. Med. Biol. 12 (1) (2012) 1250006-1-1250006-18.

[3] Y. Hoi, H. Meng, S.H. Woodward, B.R. Bendok, R.A. Hanel, L.R. Guterman, L.N. Hopkins, Effects of arterial geometry on aneurysm growth: three-di- 
mensional computational fluid dynamics study, J. Neurosurg. 101 (4) (2004) 676-681.

[4] Z.W. Chen, P. Joli, Z.O. Feng, Anisotropic hyperelastic behavior of soft biological tissues, Comput. Methods Biomech. Biomed. Eng. 18 (13) (2015) 1436-1444.

[5] J. Weiss, B. Maker, S. Govindjee, Finite element implementation of incompressible, transversely isotropic hyperelasticity, Comput. Methods Appl. Mech. Eng. 135 (1-2) (1996) 107-128.

[6] L.A. Taber, Nonlinear Theory of Elasticity: Applications in Biomechanics, World Scientific Publishing Co. Pte. Ltd., London, UK, 2004.

[7] D. Pinho, D. Bento, J. Ribeiro, R. Lima, M. Vaz, An in vitro experimental evaluation of the displacement field in an intracranial aneurysm model, in: New Trends in Mechanism and Machine Science: From Fundamentals to Industrial Applications, Springer, Guimarães, Portugal, 2015, pp. 261-268.

[8] R.O. Rodrigues, D. Pinho, D. Bento, R. Lima, J. Ribeiro, wall expansion assessment of an intracranial aneurysm model by a 3D digital image correlation system measurement, Measurement 88 (2016) 262-270.

[9] C. Yu, K. Matsumoto, S. Shida, D.J. Kim, M. Ohta, A steady flow analsys on a cerebral aneurysm model with several stents for new stent design using PIV, J. Mech. Sci. Technol. 26 (5) (2012) 1333-1340.

[10] K. Perktold, G. Rappitsch, Computer simulation of local blood flow and vessel mechanics in a compliant carotid artery bifurcation model, J. Biomech. 28 (7) (1995) 845-856.

[11] M. Oshima, R. Torii, T. Kobayashi, N. Taniguchi, K. Takagi, Finite element simulation of blood flow in the cerebral artery, Comput. Methods Appl. Mech. Eng. 191 (6-7) (2001) 661-671.

[12] P. Moireau, N. Xiao, M. Astorino, C. Figueroa, D. Chapelle, C. Taylor, J.F. Gerbeau, External tissue support and fluid-structure simulation in blood flows, Biomech. Model. Mechanobiol. 11 (1) (2012) 1-18.

[13] G. Carty, S. Chatpun, D.M. Espino, Modeling blood flow through intracranial aneurysms: a comparison of Newtonian and non-Newtonian viscosity, J. Med. Biol. Eng. 36 (2016) 396-409.

[14] G. Cattaneo, M. Schumacher, J. Wolfertz, T. Jost, S. Meckel, Combined selective cerebral hypothermia and mechanical artery recanalization in acute ischemic stroke: in vitro study of cooling performance, Am. J. Neuroradiol. (2015) Published online.

[15] E. Gussenhoven, E.J. Gussenhoven, C.E. Essed, C.T. Lancée, F. Mastik, P. Frietman, F.C. van Egmond, J. Reiber, H. Bosch, H. van Urk, J. Roelandt, N. Bom, Arterial wall characteristics determined by intravascular ultrasound imaging: an in vitro study, J. Am. Coll. Cardiol. 14 (4) (1989) 947-952.

[16] K. Perktold, G. Rappitsch, Computer simulation of local blood flow and vessel mechanics in a compliant carotid artery bifurcation model, J. Biomech. 28 (7) (1995) 845-856.

[17] M. Olufsen, C. Peskin, W. Kim, E. Pedersen, A. Nadim, J. Larsen, Numerical simulation and experimental validation of blood flow in arteries with structured-tree outflow conditions, Ann. Biomed. Eng. 28 (11) (2000) 1281-1299.

[18] M. Mehrabadi, D. Ku, C. Aidun, A continuum model for platelet transport in flowing blood based on direct numerical simulations of cellular blood flow, Ann. Biomed. Eng. 43 (6) (2015) 1410-1421.

[19] J.S. Byun, S.Y. Choi, T. Seo, The Numerical study of the hemodynamic characteristics in the patient-specific intracranial aneurysms before and after surgery, Comput. Math. Methods Med. 2016 (2016) 4384508.

[20] C.M. García-Herrera, D.J. Celentano, E.A. Herrera, Modelling and numerical simulation of the in vivo mechanical response of the ascending aortic aneurysm in Marfan syndrome, Med. Biol. Eng. Comput. 55 (2017) 419-428.

[21] L. Taber, Nonlinear Theory of Elasticity: Applications in Biomechanics, World Scientific Publishing Co. Pte. Ltd., Singapore, 2004.

[22] J. Martí, A. Oliver, J. Freixenet, R. Martí, Digital Mammography: 10th International Workshop, Springer, Institute of Informatics and Applications, University of Girona, 2010.
[23] A.A. Alhayani, J.A. Giraldo, J. Rodriguéz, J. Merodio, Computational modelling of bulging of inflated cylindrical shells applicable to aneurysm formation and propagation in arterial wall tissue, Finite Elem. Anal. Des. 73 (2013) 20-29.

[24] A.A. Alhayani, J. Rodriguéz, J. Merodio, Competition between radial expansion and axial propagation in bulging of inflated cylinders with application to aneurysms propagation in arterial wal tissue, Int. J. Eng. Sci. 85 (2014) 74-89.

[25] N. Varatharajan, A. DasGupta, Study of bifurcation in a pressurized hyperelastic membrane tube enclosed by a soft substrate, Int. J. Non-Linear Mech. 95 (2017) $233-241$.

[26] J. Merodio, D.M. Haughton, Bifurcation of thick-walled cylindrical shells and the mechanical response of arterial tissue affected by Marfanś syndrome, Mech. Res. Commun. 37 (2010) 1-6.

[27] D.M. Haughton, J. Merodio, The elasticity of arterial tissue affected by Marfan's syndrome, Mech. Res. Commun. 36 (2009) 659-668.

[28] R. Lima, S. Wada, S. Tanaka, M. Takeda, T. Ishikawai, K. Tsubota, Y. Imai, T. Yamaguchi, In vitro blood flow in a rectangular PDMS microchannel: experimental observations using a confocal micro-PIV system, Biomed. Microdevices 10 (2) (2008) 153-167.

[29] K.L. Lawrence, ANSYS Workbench Tutorial Release 14, SDC Publications, University of Texas at Arlington, 2012.

[30] X. Chen, Y. Liu, Finite Element Modeling and Simulation with ANSYS Workbench, CRC Press, 2014.

[31] Density of Glycerine-Water Solutions, 2016 accessed 21.09.2016 http://msdssearch.dow.com/PublishedLiteratureDOWCOM/dh_0032 0901b80380032282.pdf?filepath=glycerine/pdfs/noreg/115-00656. pdf\&fromPage $=$ GetDoc.

[32] The Soap and Detergent Association, Glycerine: an Overview, The Soap and Detergent Association, New York, USA, 1990.

[33] T. Proulx, Mechanics of biological systems and materials, in: Proceedings of 2011 Annual Conference on Experimental and Applied Mechanics, Springer, USA, 2011.

[34] A.K. Bhowmick, M. Hall, H. Benarey, Rubber Products Manufacturing Technology, CRC Press, New York, USA, 1994.

[35] B. Donald, Practical Stress Analysis With Finite Elements, Glasnevin Publishing, Ireland, 2007.

[36] E. Austrell, L. Kari, Constitutive models for rubber IV, in: Proceedings of fourth European Conference on Constitutive Models for Rubber, London, UK, 2005.

[37] A. Amin, M. Alam, Y. Okui, An improved hyperelasticity relation in modeling viscoelasticity response of natural and high damping rubbers in compression: experiments, parameter identification and numerical verification, Mech. Mater. 34 (2) (2002) 75-95.

[38] D. Lin, D. Shreiber, E. Dimitriadis, F. Horkay, Spherical indentation of soft matter beyond the Hertzian regime: numerical and experimental validation of hyperelastic models, Biomech. Model. Mechanobiol. 8 (5) (2009) 345-358.

[39] R.B. Bird, R.C. Armstrong, O. Hassager, Dynamic of Polymeric liquids, Vol.1, John Willey \& Sons, New York, USA, 1987.

[40] Y.A. Çengel, A.J. Ghajar, Heat and Mass Transfer, Fourth Ed., Mc. Graw Hill, USA, 2012.

[41] Y. Bazilevs, M.C. Hsu, Y. Zhang, W. Wang, T. Kvamsdal, S. Hentschel, J.G. Isaksen, Computational vascular fluid-structure interaction: methodology and application to cerebral aneurysms, Biomech. Model. Mechanobiol. 9 (4) (2010) 481-498.

[42] J. Merodio, R.W. Ogden, J. Rodríguez, The influence of residual stress on finite deformation elastic response, Int. J. Non-Linear Mech. 56 (2013) 43-49. 